\title{
Motivating participation in social computing applications: a user modeling perspective
}

\author{
Julita Vassileva
}

Received: 16 November 2010 / Accepted in revised form: 6 April 2011 /

Published online: 10 March 2012

(C) Springer Science+Business Media B.V. 2012

\begin{abstract}
The explosive growth of Web-based social applications over the last 10 years has led people to engage in online communities for various purposes: to work, learn, play, share time and mementos with friends and family and engage in public action. Social Computing Applications (SCA) allow users to discuss various topics in online forums, share their thoughts in blogs, share photos, videos, bookmarks, and connect with friends through social networks. Yet, the design of successful social applications that attract and sustain active contribution by their users still remains more of an art than a science. My research over the last 10 years has been based on the hypothesis that it is possible to incorporate mechanisms and tools in the design of the social application that can motivate users to participate, and more generally, to change their behavior in a desirable way, which is beneficial for the community. Since different people are motivated by different things, it can be expected that personalizing the incentives and the way the rewards are presented to the individual, would be beneficial. Also since communities have different needs in different phases of their existence, it is necessary to model the changing needs of communities and adapt the incentive mechanisms accordingly, to attract the kind of contributions that are beneficial. Therefore User and Group (Community) Modeling is an important area in the design of incentive mechanisms. This paper presents an overview of different approaches to motivate users to participate. These approaches are based on various theories from the area of social psychology and behavioral economics and involve rewards mechanisms, reputation, open group user modeling, and social visualization.
\end{abstract}

\footnotetext{
J. Vassileva $(\varangle)$

Computer Science Department, University of Saskatchewan, 176 Thorvaldson Bldg.,

110 Science Place, Saskatoon, SK S7N 5C9, Canada

e-mail: jiv@cs.usask.ca

URL: http://julita.usask.ca; http://www.madmuc.com
} 
Future trends are outlined towards convergence with the areas of persuasive systems design, adaptive/personalized systems, and intelligent social learning environments.

Keywords Social computing · Participation · Motivation · Persuasion · Gamification · Open user models · Group user models · Reflection · Adaptive incentive mechanism · Incentives · Mechanism design

\section{Introduction}

The explosive growth of Web-based social applications over the last 10 years has led people to engage in online communities for various purposes: to work, to learn, to share time and mementos with friends and family and engage in public action. Social Computing Applications (SCA) allow users to discuss various topics in online forums, share their thoughts in blogs, share photos, videos, bookmarks, and connect with friends through social networks. Yet, the design of successful SCA that attract and sustain active contribution by their users, still remains more of an art than a science. For every successful one, there are thousands that have failed. There has been research over the last 10 years in the areas of human-computer interaction and the social sciences on how and why people engage in large successful SCA. Advances have been made (mostly by the Web 2.0 industry) in developing technologies for social infrastructures for online communities and social networks, e.g. Wordpress, Tumblr, etc. Practical guides and even software patterns have appeared on how to design social interfaces to attract participation (Kim 2000; Porter 2008,Crumlish and Malone 2010). Yet, these "best practices" and "lessons from the trenches" make sense in retrospect looking at successful SCA, but there are no general recipes or methodologies of how to develop new SCA from scratch.

In the past 10 years, the research community has been searching for a methodology for attracting participation by designing reward mechanisms (incentive mechanisms) inspired by different behavioral science theories, using a trial and error approach. The hypothesis is that it is possible to incorporate in the design of the social application incentive mechanisms and interventions that can motivate users to participate, and more generally, to change their behavior in a desirable way, which is beneficial for the community.

Why is this area relevant to User Modeling and Personalization? It is well known that different people are motivated by different things in different ways, so it can be expected that personalizing the incentives and the way the rewards are presented to the individual would increase the effect of the incentives on their motivation. Also groups of users and online communities have different needs of contributions in different phases of their existence (Jones and Rafaeli 1999). For example, in the beginning, any contributions help the community to "take off", but later, high quality contributions are important and mechanisms to emphasize high-quality contributions become a necessity. Modeling the changing needs of communities and adapting the incentive mechanisms accordingly can help attract the kind of contributions when they are most needed. Therefore User and Group (Community) Modeling is an area that can provide valuable insights and techniques in the design of adaptive incentive mechanisms for participation. 
This article presents an overview of different approaches to motivate users to participate and contribute to online communities, with some of the main problems illustrated with systems designed and evaluated in the ARIES and MADMUC Labs at the University of Saskatchewan over the last 10 years. These approaches cover a spectrum of incentive mechanisms: from extrinsic, through social, to intrinsic. Some of them are based on different theories from the area of social psychology and behavioral economics and involve economics rewards mechanisms, reputation, open group user modeling, and social visualization. The next section presents some of the main challenges in design of motivation mechanisms and approaches that address them. Then directions of future development and convergence with other active research areas are presented.

\section{Theories inspire design approaches to motivating user participation}

Why people act in particular ways is a fundamental question that has been in the focus of economists and psychologists since these disciplines exist. While space limitations do not permit presenting a detailed overview of theories of motivation, the following brief overview aims to highlight the main perspectives on motivation that exist in literature. This will help to identify the problems in designing social infrastructures motivating users to participate that are discussed in this section. More comprehensive reviews of theories of motivation and how they have been used in design of social systems can be found in (Ling et al. 2005) and (Kraut and Resnick, forthcoming).

\subsection{Economic view of motivation: example design and challenges}

Classical Economics approaches the issue of motivation by assuming that people are rational agents who act to maximize their utility (payoff) in a world where behaviours have certain payoffs (negative or positive). Thus to make people behave in particular way, one needs to create an appropriate system of incentives (rewards) for the desirable behaviours. Incentive mechanism design (also called just "mechanism design") is a very active area of research in mathematical economics and game theory. The goal is to design rules of encounter that, when followed by the participants, will ensure that the overall system fulfils a particular goal, or fits a set of criteria, e.g. optimize the joint welfare for all participants, ensure fair chance for them to maximize their utilities, or simply to maximize the utility of the owner of the system. The diversity of motivations that may exist among the members of the community is not taken into account; they are all utility maximizers and follow the same rules. The payoffs for particular actions may be subjectively different, i.e. each participant may have her own unique utility function. Most of the applications of mechanism design are in tightly constrained systems, auctions. Approaching the problem of motivating participation in a community as an economic mechanism design emphasizes the benefit of the system or community as a whole, rather than that of the individual users.

\subsubsection{Economic mechanism design (Marketplace)}

An example of an economic mechanism based on virtual currency can be found in a peer-help community called "I-Help" (Greer et al. 1998). The mechanism regulated 
the demand and supply of help in the community (Vassileva et al. 1999; Kostuik and Vassileva 1999). Students could be buyers and/or sellers of help on various questions/topics. Virtual currency was used to complete trades. The price depended on the scarcity of helpers competent in answering a question on a given topic at the moment of the request. The accumulated currency by students was exchanged at the end of the term for something of real-world value.

\subsubsection{Challenge in creating an appropriate market model for the community}

While introducing currency and market is fairly straightforward, challenges arise, related to the specifics of the community in which the market is introduced (Greer et al. 2001). I-Help, for example, was a learning community, where the main traded good was help.

Help is quite a different good from tangible goods (such as those traded on eBay). In any group there are weaker students who mostly need help and are rarely able to provide help to others. In a pure market-based system, these students are likely to become "bankrupt", i.e., unable to buy help anymore, thus being shut out of the system.

In I-Help, of course, such an outcome was undesirable, since the main goal of the system was to increase the knowledge of all students by creating incentives to students who had knowledge to give help and by giving a fair chance to everyone to buy help. Therefore, a "social welfare system" had to be introduced. However, providing a fresh supply of virtual money (e.g., a weekly allowance) complicated the economy significantly, leading to inflation. The total amount of currency was no longer fixed but could grow unlimited which made it hard to match adequately the virtual currency earned by the students with real world rewards. An economic approach to this problem, by introducing taxation on earnings, would have complicated further the mechanism and would likely have been de-motivational for active helpers. Putting a cap on the earnings of active helpers would have also been a disincentive to continue helping after they had reached the cap. The pedagogical goal of the system was to encourage students to always help, even if they were the top helpers in the community, since one learns more by helping than by receiving help. This could not be achieved with the economic model of I-Help, where the currency was injected in the system from outside, rather than generated from within the community, while knowledge was a positive externality that was generated from within the community during help sessions, but formally unaccounted for in the model.

\subsubsection{Challenge in designing the user view of the mechanism}

Another challenge arising in the system design is how much of the underlying economic model should be revealed to the user. This is a general problem with all incentive mechanisms, whether they are based on a market model, a game-like system where users collect points or earn reputation (as discussed in the next section), or on a psychological theory of motivation. In I-Help, since the general purpose of the system was to facilitate learning, it was important to keep the students' attention focused on learning, rather than on trading help and earning currency. For this reason, instead of having the users explicitly trading for help, as in Google Answers, the economic 
transactions (negotiating for the price and dealing with the payments) were delegated to the software infrastructure (the personal agents of the uses), who maintained models of user preferences with respect to price, availability, topics of competence etc. For details on the negotiation approaches used by the agents, see (Mudgal and Vassileva 2000; Winoto et al. 2005).

\subsubsection{Challenge in adapting at run time the parameters of the mechanism}

Another challenge is that often the mechanism is a part of a dynamic system and it often requires user input to adapt the rewards dynamically to the situation at hand. For example, in I-Help the mechanism did not consider the quality of help exchanged in the price negotiation, but only the help demand/supply ratio at the moment. It could happen that after a help session started, one of the parties discovered that the session was a waste of time. The system, however, allowed students to quit a session at any time to avoid being charged for useless chatter. A time-meter mode of payment (similar to a telephone call), i.e., the price per minute of help was negotiated by the agents, instead of a total price for the session, thus allowing any partner to interrupt the chat-session if they felt they were not getting value from it.

An alternative solution, more typical of current online communities is to collect feedback (ratings) after the session by both partners and compute reputation for each helper and helpee (Wang and Vassileva 2003, 2004). This would allow the agents of users with high reputation to charge higher prices for their services and would have also provided an incentive for users to give good help.

\subsubsection{Other social applications using market mechanisms}

Many early (between 2000 and 2006) multi-user systems used market mechanisms and virtual currency, cashed in either real dollars, in better performance, or in reputation. One can find a variety of economic models as theoretical proposals in the area of multi-agent systems and peer-to-peer (p2p) systems, e.g. the use of micro-payments to motivate contributions to (Golle et al. 2001). In some p2p communities, such as BitTorrent, the accumulated micro-payments are earned by sharing more files, staying online, offering good bandwidth and are "cashed" in better performance in terms of download speed.

Two large-scale communities similar to I-Help, but not in educational context, are Google Answers (Rafaeli et al. 2007), and more recently, Yahoo Answers. Google Answers operated between 2001 and 2006 and was based on a market model using real dollars. However, research into the user motivations in these communities, e.g. (Rafaeli et al.2007) shows that the participation of experts is associated with a hybrid of economic and social motivators, such as "star" ratings, and user feedback on answers. The monetary rewards were responsible for the demise of Google Answers, since the community was ridden by gamers trying to exploit the system and make money, while not providing any valuable answers and causing a lot of user complaints.

To avoid following into Google Answer's steps, Yahoo Answers uses a modified currency mechanism that rewards active users with a range of honor badges ("power users", "top contributor", etc.) that are visible to other users and represent their 
reputation in the community. This kind of mechanism is in line of modern behavioral economics, and incentive mechanism designs along these theories will be discussed in the next section. Other authors, e.g. (Hsieh et al. 2010), have analysed more recently the applying monetary incentives in question-answering systems.

\subsection{Behavioral economics view of motivation}

In contrast to classical economics, behavioral economics views people as irrational and investigates, most often experimentally, the social, cognitive and emotional factors in understanding the economic decisions of individuals. Many findings of Behavioral Economics relate to why people make certain choices and what drives or motivates people's behaviors, showing that many theoretically sound economic mechanisms are not psychologically valid and fail when tried with real users (Ariely 2008). Ernst Fehr and his colleagues (Fehr et al. 1998), and others (e.g. Armin Falk, Matthew Rabin) studied psychological phenomena, such as "fairness", "inequity aversion", and "reciprocal altruism", which put in question the classical economics assumption of "perfect selfishness." Other studies have shown that the introduction of extrinsic rewards undermines or entirely replaces intrinsic motivation (Lepper et al. 1973). The area became popular with the recent book "Predictably Irrational" by Dan Ariely (2008). The ideas from behavioral economics in the context of proliferating social networking sites, smart phones and pads with sensors, gave rise to user engagement design approaches aiming to increase participation on SCA (Crumlish and Malone 2010; Porter 2008). One major direction is the so called "Gamification" of SCA, or introducing elements of game in the design of the user interaction with SCA.

\subsubsection{Gamifiation and game mechanics}

"Gamification" is "the integration of Game Mechanics in non-game environments to increase audience engagement, loyalty and fun" (www.gamification.org, for academic references see Deterding et al. 2011a,b). The related area of practical expertise called "Game Mechanics" has accumulated a number of patterns, rules and feedback loops, that are motivational, create user engagement and loyalty and can be applied to develop game-like elements in virtually any application or community. Examples of the most commonly used patterns are: ownership (allowing the user/player to own things, such as points, tokens, badges, since it creates loyalty to the application, game or community); achievements (providing a virtual or physical representation of having accomplished something that can be easy, difficult, surprising, funny, and accomplished alone or as a group), status (computing and displaying a rank or level of a user), community collaboration and quests (posing challenges to the users related to time-limit or competition, that can be resolved by working together).

Reputation has been used in online communities to motivate participation for a long time. Slashdot pioneered this approach by introducing the notion of "karma" in the mid 1990ies to reward users who gave good comments with visibility and power in the community. Currently most social sites provide ways for users to build their reputation based on the ratings received by their contributions. The most prominent examples 
are eBay's seller and buyer reputation ratings and Amazon's reviewer ratings. Yet, designing successful reputation schemes can be quite challenging.

\subsubsection{Challenge in selecting what user actions to reward with reputation and status}

The difference between status and reputation is that while status can be earned by the user in isolation, by performing certain actions, reputation is based on the opinion of other users about the user or her contribution. Reputation can be developed, for example, by posting articles that earn very high ratings. Rock groups and celebrities on Twitter measure their reputation by the number of fans/followers. Users on Facebook keep track of their reputation by the number of friends they have. However, the term "reputation" has been used often interchangeably with status. For example, Amazon calculates what they call "reputation" of book reviewers based on the number or reviews they have written (this would be their status in our definition) and the ratings these reviews have obtained (this would be their reputation according to our definition).

It is not straightforward to select for a particular community which user actions should be rewarded with status and/or reputation, what privileges should be granted, and for what levels of status and/or reputations.

Next, this challenge will be illustrated with an example from the design of Comtella (Vassileva 2002) — an online community infrastructure based on a P2P architecture to support sharing files with academic papers among a research group or class, thus something like a predecessor of academic paper sharing sites like CiteULike, Zotero or Mendeley.

The first incentive mechanism applied in Comtella (Bretzke and Vassileva 2003; Cheng and Vassileva 2005a) rewarded users with points for actions that were beneficial for the community (contributing new papers, downloading papers from others and making them available for sharing with others). These were actions that the user had full control of and did not reflect the opinion of other users of the user's actions. Thus the reward was called "membership level/status" rather than "reputation". Each user was classified, depending on the accumulated points into one of three different status levels (gold, silver, bronze). Different status levels implied different privileges (e.g. interface appearance, number of ratings to give out). The results of the evaluation of this mechanism showed a significant but short-term increase of participation. There were attempts by some users to game the system, by performing unreasonably high numbers of the rewarded actions (Cheng and Vassileva 2005a). Since the quality of the contributions was not evaluated, the users' participation in the system deteriorated due to the overwhelming amount of low-quality contributions and the resulting cognitive overload (Jones and Rafaeli 1999).

Other online communities define status-levels based on other criteria, which are harder to game, for example, since how long the user has been a member of the community (i.e. "member since"). However, this definition of status can be used only for long-term communities, and may not be motivational for new members.

If increasing participation was the only goal, the first Comtella incentive mechanism based on status was quite effective. However, due to the problem of gaming, that 
could not be tackled without introducing a measure of quality of contribution in the mechanism, so reputation had to be introduced in the next version of Comella.

The reputation of a user was calculated as a function of the ratings received by the user's contributions by other users. However, incentivizing users to rate the contributions of others is not easy; this is again a problem of increasing participation (of different kind), encountered by all systems that rely on user ratings (e.g. recommender systems). To encourage users to rate, a market-based model with virtual currency (c-points) was introduced. The user could earn c-points by rating a resource and could spent them to promote her own contributions (like Google's sponsored links). The currency model was very successful in stimulating ratings, and resulted in twice higher amount of ratings generated by the experimental group versus the control group in a controlled experiment. With many ratings, the computation of user reputation became more accurate.

Unfortunately, in most real communities and applications, there are also general system goals, similar to those existing in the area of market design. For example, it may be desirable that the user contributions follow a particular time pattern since usually the early contributions are more important than late contributions, since they set the tone of future contributions, provoke users to respond or share their own contributions. Later on, as the volume of contributions increases, it becomes important to get users to rate the contributions of others, so that good resources can be found more easily. Also high quality contributions should be rewarded at any time. Therefore, a need arises to create dynamic incentives that "orchestrate" the individual user behaviors to produce a harmonic overall behavior of the system. For this the patterns of Game Mechanics become insufficient.

\subsubsection{Dynamic, adaptive and personalized rewards and reputation}

No general theories or guidelines exist for designing mechanisms with dynamic rewards. They are crafted according to the specific needs of the community. As an example, the second version of the Comtella incentive mechanism is presented here.

The new incentive mechanism aimed to encourage contribution of links to highquality articles, to discourage excessive contribution and to encourage timely contributions (Cheng and Vassileva 2005b). The rewards for each participative action (contributing papers and contributing ratings) were increased or decreased dynamically according to the individual's reputation for contributing high quality papers and high quality ratings. Since Comtella was deployed in an educational context, where students were sharing articles related to the weekly topics discussed in their class, one of the overall goals was to ensure that students shared their articles early in the week, so that there was time for their colleagues to read, rate and comment them. So the weight of each action depended on the day of the week and on the number of resources that had been already contributed by the community. To prevent over-contributions by students who might have tried to game the system to achieve high status, there was also a personal cap on the number of rewarded contributions which depended on the quality of the previous contributions by the user and the desired number of contributions for the week for the entire community, set by the instructor. In this way the status 
of the user was calculated based on dynamic, adaptive rewards that took into account a model of the community's needs and the model of the individual contributions of each user.

The results of a controlled study evaluation with 21 students showed that the mechanism was very effective and stimulated exactly the behavior that was desired. The conclusion was that a mechanism with adaptive rewards to the individual patterns of contribution and to the needs of the community could orchestrate/ conduct the desired patterns of behavior in the individual users, leading to a sustainable level and higher quality of contributions. More details about the incentive mechanism design and the study can be found in (Cheng and Vassileva 2006).

To our best knowledge, there haven't been other incentive mechanisms of comparable complexity proposed in research literature. However, it is well possible that such mechanisms have been implemented in real systems, but never revealed. Generally, most successful large scale communities do not reveal details about the incentive mechanisms that are deployed, since otherwise they would be challenged by gamers.

\subsubsection{Critical view of gamification}

As explained above, the idea of adding game elements in non-game applications and social sites has a lot of potential. Recently, however, some influential bloggers (McDonald 2010; Radia 2010; Wu 2011), have been critical to the gamification trend, pointing out while most of the current gamified sites make their users collect points for trivial actions, thus devaluing the rewards. McDonald predicts on her blog that soon it will be "Game over" for this type of applications. The reason is that the ubiquitous points gathering is based on a simplistic economic and behaviourist model and is leads to a motivation only for a short time. The current hype of gamification will unavoidably disincentivize the most creative elite users, who are most valuable for any community or social application. Both McDonald and Radia emphasize the need of developing different types of games that foster a sense of achievement rather than points and badges, that create intrinsic motivations rather than replacing them with extrinsic rewards (points and badges), and that reintroduce genuine play and genuine delight. As Deterling puts it (Bozarth 2011):

... we play games, because we inherently enjoy the activity. If you look further at what makes an activity inherently enjoyable, then you see that games deliver on all three things in the current major theory of intrinsic motivation, self-determination theory: they give you experiences of competence, autonomy, and relatedness.

It seems that despite the foray into designing simplistic rewards mechanisms and the gamification of social sites, designers are turning again to the fundamental questions about what motivates people, an area that has been actively studied by classical disciplines like Psychology, and newer ones, like Social Psychology, Organziational Behaviour, Media Studies. 


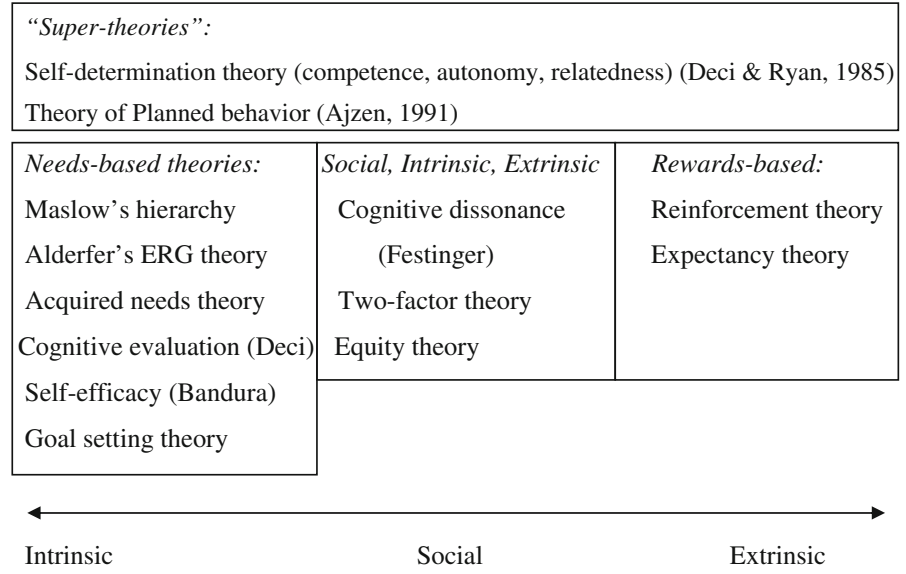

Fig. 1 A spectrum of motivation theories in psychology

2.3 Theories of motivation and approaches inspired by them

\subsubsection{Motivation theories from psychology}

Motivation has been studied extensively in the area of Psychology, where a wealth of theories of motivation has been developed over the last 100 years. The focus of these theories is the individual and her experiences with the environment and other individuals, or society as a whole. It is impossible to provide here an overview of any depth of the existing theories of motivation, due to their sheer number. To navigate among the spectrum of theories, we will consider a distinction that could be considered as a watershed between two clusters of influential psychological theories of motivation (Fig. 1). This is the distinction between extrinsic motivation (from outside, driven by external rewards or pressure from the environment and other individuals) and intrinsic motivation (from within, driven by interest or enjoyment that the individual experiences from the activity). This classification serves the purposes of this article to help the reader navigate in the theories since the three positions of the spectrum can be found in existing design patterns. However, we do not claim that this classification has any larger validity. In fact the distinction between the categories is quite blurred and there are researchers (e.g. Reiss 2004) who question even the distinction between intrinsic and extrinsic motivation, emphasizing that it is all a matter of individual difference. Reiss (2004) proposes a theory of 16 basic desires, which can exist simultaneously, or with different strength at different times in different individuals. Some theories, e.g. the self-determination theory (Deci and Ryan 1985) and the theory of planned behavior (Ajzen 1991) encompass both intrinsic and extrinsic motivations.

Extrinsic motivation (rewards) is the focus of Skinner's reinforcement theory and the expectancy theory. On the other side of the spectrum, intrinsic motivation are in the focus of the needs-based theories of Maslow, Alderfer's ERG Theory, the acquired needs theory, as well as Bandura (1997) self-efficacy theory and the goal setting theory 
(Latham and Locke 2002). In the middle of the spectrum, the Herzberg's two factor theory, the equity theory and the cognitive evaluation theory consider the interplay of intrinsic, extrinsic and social motivators.

\subsubsection{Explaining why certain game-design patterns work}

Many of the abovementioned theories can explain the motivational effect of the game design patterns mentioned previously. Clearly all these patterns provide extrinsic rewards for the users which, according to extrinsic motivation theories, should provide motivation in the users to perform the actions or behaviours that lead to rewards (e.g. collect points, badges). Theories in the middle of the spectrum explain the motivational effect of reputation, which has meaning only in a social environment. On the other side, since different people consider different things as rewarding, depending on their intrinsic needs, values and goals, the theories on the Intrinsic end of the spectrum and those in the Social category explain the different possible needs that people have. For example, the motivational effect of reputation and status can be explained by all needs-based theories, like Maslow's, Alderfer's ERG and the Acquired Needs theories, Social Psychology, which all point out to the need of humans to socialize and seek social recognition and status. It can be also explained by Bandura's self-efficacy theory (1997), since usually social status and reputation is a result of recognized mastery, which is one of the four major sources of self-efficacy. A visible reputation in a group sets conditions for another source of self-efficacy, social modeling, or witnessing people successfully completing tasks or demonstrating mastery.

For example, let's focus on the theory of social comparison (Festinger 1954). This theory was generalized a few years later by Festinger and became part of his Theory of Cognitive Dissonance. It states that people tend to compare themselves with others, who they perceive as similar to them, in order to evaluate or enhance some aspects of the self (Suls et al. 2002). Whether the social comparison serves a self-enhancement function depends on whether the comparer assimilates or contrasts his or her self relative to superior or inferior ones. Two processes can be observed: assimilation, facilitated by the belief that one can obtain the same status as the target (the role-model), and contrast - comparison with dissimilar ones to enhance or protect the subjective well-being and thereby satisfy the self-enhancement motive.

The Social Comparison Theory can explain the motivational effect of the leaderboard pattern in game mechanics and has been the inspiration for design of incentive mechanisms in several research projects. In Comtella, we sought to encourage upward assimilation by visualizing the status and reputation of users using a star-sky metaphor (Bretzke and Vassileva 2003). Each user was shown as a star on a night sky with colour - corresponding to the status (gold, silver, bronze), brightness — corresponding to the reputation of the user, and size-corresponding to the number of shared papers (Sun and Vassileva 2006). We found that many users checked their reputation status in the visualization, and that users who checked their status more frequently contributed more. Similar results have been reported by other authors proposing similar incentive mechanisms based on reputation status and social comparison. For example, (Farzan et al. 2008) show 2 times increase of contributions in the Beehive system, using a 
reputation-based approach similar to Comtella. Others (Chen et al. 2007; Harper et al. 2007) show 5 times increase in the number of monthly movie ratings when shown the median of user contribution in a monthly newsletter. It seems that social comparison can be used as a powerful incentive and effectively increase contributions to online communities.

\subsubsection{Theories of motivation from other areas}

More recently, newer areas of social science, such as Social Psychology, Educational Psychology, Organizational Science, and Sociology (Media Studies) have contributed more theories focusing on motivation in particular types of environments. For example, in workplace and organizational context, the Collective Effort Model explains the motivation for contributing to teamwork. Other theories, such as the Common Identity Theory, and the Common Bond theory also explain motivations for contribution to a group. In the area of Educational Psychology, the Self-efficacy Theory and Goal-setting Theory explain and predict motivation to learn. In the area of Social Psychology important theories have been developed to explain persuasion with application in marketing (advertisement) and behavioural change (e.g. encourage physical fitness, or smoking cessation): The Trans-theoretical model, the Social Comparison Theory and the more general Theory of Cognitive Dissonance, and the Theory of Discrete Emotions (Cialdini 2001). Theories specific to human motivation for consuming or being involved in particular media have appeared in the area of media studies, e.g. the Needs and Gratifications theory (Katz et al. 1973) which explains the users motivation to interact with particular media with their inherent needs for entertainment, information and which can be hampered by irritation.

These newer theories have more emphasis on intrinsic motivation and therefore hold a promise to inspire newer motivational patterns and incentive mechanisms that emphasize achievement, altruism and genuine delight of gaming (in contrast to the currently used gamification patterns). For example, Rashid et al. (2006) proposed an approach to increase intrinsic motivation based on emphasizing the value of the user's contribution.

\subsection{4 "Gentle" approaches appealing to intrinsic motivation and reciprocity}

Social comparison, status and reputation can clearly provide a strong motivation for participation for a large part of the users. However, there are users that are immune to reputational incentives, but are willing to contribute to a cause they believe in, to help their friends, or to make a difference through their actions to the benefit of the community. Theories like the Common Identity Theory and Common Bond from the area of Organizational Behavior explain such motivations. The common identity theory makes predictions about the causes and consequences of people's attachment to the group as a whole, while the common bond theory makes predictions about the causes and consequences of people's attachment to individual group members. The causes of common identity are social categorization, interdependence and intergroup comparison (Turner 1985; Turner et al. 1987). After studying existing discussion forums, CMU researchers (Ren et al. 2007) suggest a set of design suggestions to encourage 
users in communities based on either of these two principles to integrate better and be more productive members:

Identity-based communities should have clear mission statements and policies to keep conversation on topic, can tolerate anonymity and large numbers of participants, and can conduct all communication in public forums. By contrast, bond-based communities should phrase their mission statements to encourage members to engage in and to tolerate conversations on wide-ranging topics, and would improve if the numbers of participants were limited, and if they had mechanisms for private communication and identifying members. (Ren et al. 2007)

We also explored motivational approaches aiming to appeal to the users' intrinsic motivations. One of these approaches (Sahib and Vassileva 2009) was inspired by the Common Identity Theory, and the other two approaches (Webster and Vassileva 2006; Raghavun and Vassileva 2011) were inspired by the Common-Bond Theory and the Theory of Reciprocation (Fehr et al. 1998). While the evaluation results of our approaches based on the Common Identity and Common-Bond Theory were inconclusive, the reciprocation-inspired mechanism (Webster and Vassileva 2006) was successful in engaging users to develop reciprocal relationships among each other. The theory of reciprocation (Fehr et al. 1998), states that people generally tend to return favors received from others. We designed a visualization that shows the relationship between the user (viewer) and the other users with respect to the two sides of the relationship: how much the other user tends to view the posts of the viewer and how much the viewer tends to view the posts of the other user. We expected that realizing who views their posts would trigger users to reciprocate by viewing / rating their posts in return. A controlled study with nearly 80 users showed that the reciprocity visualization stimulated the build-up of a significantly higher number of reciprocal relationships in the experimental group than in the control group. More details are provided in (Webster and Vassileva 2006).

\subsection{Summary}

In summary, this section gave a broad overview of existing approaches, design patterns and theories related to motivating participation in social applications. There exist simple approaches and design patterns that have been shown to successfully engage users, and are widely applied in the gamification of social applications. Yet, these approaches are only able to ensure that users perform certain actions and are unable to steer the social system towards a desirable overall behavior. An approach using adaptive dynamic incentives guided by community needs model and individual user models was presented briefly, that was demonstrated to orchestrate a particular overall time- and activity pattern beneficial for the entire community. Finally, some of the most often cited theories of motivation that have inspired research on design of motivational patterns and mechanisms, emphasizing those providing intrinsic and social motivation were presented. The next section makes an attempt to outline future trends in the area. 


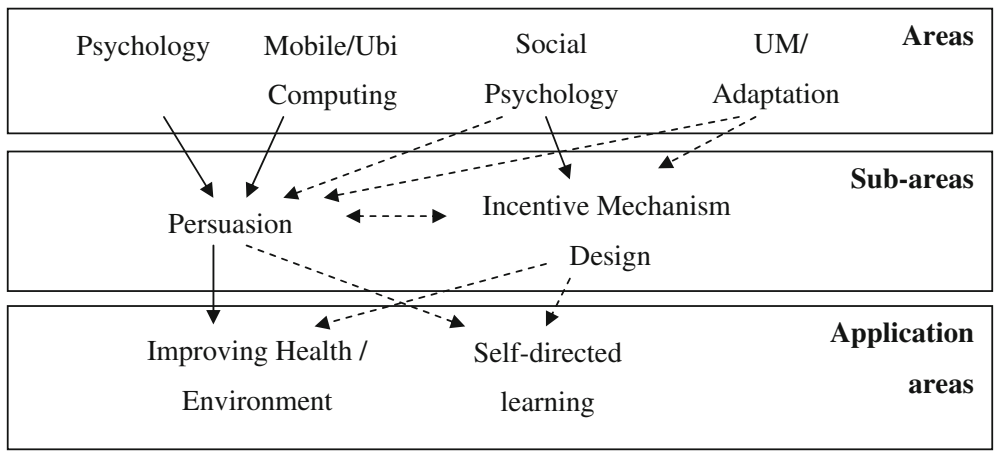

Fig. 2 The existing (solid arrows) and possible future influences (dotted arrows) between areas, sub-areas and applications

\section{Future trends}

It is hard to predict the future for an area so closely connected to one of the fastest growing areas of Computer Science - Social Computing. Yet, it seems safe to outline several trends.

One clear trend, that has been ongoing for years now, is exploring further which of the numerous contemporary theories of motivation in the areas of social psychology and behavioral economics can be usefully applied in designing reward mechanisms for particular types of communities.

A number of other trends concern the influences among different fields and their tendencies for convergence (see Fig. 2):

One trend seems to be a convergence between the area of incentive mechanism design and the quickly growing area of design of persuasion systems.

Parallels exist between the design of user-adaptive systems and incentive mechanism design, which can inspire more work on designing personalized incentives and social visualizations using approaches developed in the area of open user modeling.

Parallels exist also between the design of adaptive learning environments and the incentive mechanism design, and a cross-fertilization may bring interesting insights and useful new techniques to benefit both fields.

\subsection{Exploring designs of mechanisms inspired by theories of motivation}

A number of theories of motivation exist in the literature, which could possibly inspire the design of incentive mechanisms for participation in online communities (Ling et al. 2005). Apart from the Social Comparison Theory and Theory of Discrete Emotions, mentioned in the previous section, researchers have started exploring systematically approaches based on theories from the area of Organizational Behavior, e.g. the Common Identity Theory (Ren et al. 2007), the Uses and Gratifications Theory, the Organizational Commitment Theories (Lampe et al. 2010). However, since theories of motivation have been developed in many different fields, in addition to Psychology and Social Psychology, e.g. in Educational Psychology, Organizational Science, 
Behavioral Economics, the supply of relevant-looking theories is likely to warrant many years of research.

There are certain challenges ahead: incentive mechanisms that are applied in a real community are rarely grounded on a single theory; usually they rely on motivations along two or three theories in combination. It is therefore hard to attribute success or failure to a particular theory. It is also very hard to control external factors that can influence participation in unpredictable ways, e.g. certain external events that may fascinate the community and trigger unexpected bursts of participation, software or system failures that can kill participation if they happen in a critical phase at the start. Moreover, the success of particular incentive mechanism design in one community does not guarantee that the same mechanism will be successful in another community, so there will be a great need of repeated studies in different conditions to confirm earlier findings.

To avoid the long and laborious experimental design with many uncontrolled variables in real online communities, there have been already attempts to use computer simulation (especially Multi-Agent Simulation) to predict the effect of specific incentive mechanism in a community with a certain distribution of user types in the population (Mao et al. 2007; Ren and Kraut 2010). It would be natural to see also attempts for theoretical modeling and formalization for very specific mechanisms under narrow constrains, along the lines of game theoretic mechanism design.

\subsection{Convergence of incentive mechanism design, persuasion and personalization}

The area of Persuasion, also called "Captology" by its creator, BJ Fogg (2003) has been developing rapidly over the last few years, with the proliferation of smart phones that can extend the scope of interventions in real contexts. The focus of most researchers in the area of Persuasion is on influencing people to change their motivation, attitudes, and real world behaviors for their own benefit (e.g. eat healthier, exercise more) or for the benefit of the environment and their real community (save electricity, share rides, etc.). A great number of theories of motivation from the fields of Psychology and Social Psychology have been used as a theoretical underpinning of persuasive interventions in various domains, mostly health-related (healthy eating, exercise or smoke-cessation). Some of these theories are (Consolvo et al. 2009): the Goal-Setting theory, the Reinforcement theory, Equity theory, Expectancy theory, Activation theory, Affect perseverance, Attribution theory, Cognitive Dissonance, Self-Efficacy theory (Bandura 1997), Control (Choice) theory, Drive (or Drive Reduction) theory, Endowed Progress theory, Cognitive Evaluation theory, Reactance theory, Positive Psychology theory, and theoretical models of motivation, such as the Collective Effort Model, the Trans-Theoretical Model, and others. Most of these theories and models are related to the motivation of an individual to act in their environment, which implicitly includes other people and communities, but they do not explicitly address the motivation of a person to contribute to a community.

A level of personalization is usually present in most persuasive approaches, e.g. the content of messages or interventions shown to the user changes depending on sensor data (number of steps made during the day, blood sugar level or number of heart-beats 
per minute, etc.). However, as Nguyen and Masthoff (2010) argued, there hasn't been much work on selecting or adapting the persuasion approach and the type of interventions to the user, which is surprising, since the most effective behavioral determinants, behavior change techniques and modes of delivery depend on the individual. Yet, we have seen a growing interest towards the area of persuasion among user modeling researchers (Colineau and Paris 2010; Berkovsky et al. 2010; Freyne and Berkovsky 2010; Kimani et al. 2010).

The interventions (e.g. reminders, visualizations, personal agent's interventions) used by persuasion applications typically address the user in isolation, based on predicted by the theory reaction of the user according to the specific theory on which the approach is based. Recently, however, persuasion researchers have started to include the user's friends and family as actors in the motivational interventions (Lin et al. 2006; Khaled et al. 2006) and generally have sought to engage the user's social network as a source of persuasion (Munson et al. 2010). This can be seen as a trend towards convergence with the area of incentive mechanism design for communities.

So it seems that in the future there will be a stronger cross-fertilization between the areas of Persuasion and Incentive Mechanism Design: on one hand-expanding the range of motivations to contribute not only to online communities, but also to real ones, incentivizing users to engage in volunteering and civic action, and on the other-mobilizing the user's community and social network to help users achieve their personal goals in real life, e.g. eat healthier, quit smoking, exercise more, and engage more in common activities with their friends and family.

\subsection{Convergence of the areas of user-adaptive systems design and incentive mechanism design}

The purpose of incentive mechanisms is to change the state of the user (her goals, beliefs, motivations), i.e. to adapt the individual user to the benefit of the overall system or community. This is the opposite of the purpose of user-adaptive environments, which is to adapt the system to the needs of the individual user (see Table 1). Most work on incentive mechanism design can be viewed as orthogonal to personalization, since it based on the assumption that a community needs not personalized, but common rules for rewarding user behavior, to ensure fairness.

However, stepping up from the individual (micro) to the community (macro) level, an incentive mechanism can be viewed an adaptation mechanism towards the behavior of a community of users. It monitors the actions of the community represented in a community model, or in a collection of individual user models, and makes adaptations to the interface, information layout, or functionality of the community, to respond to the changes in the user model according to some predefined goal (e.g. maximizing participation). The parallels between adaptation mechanism in a personalized environment and an incentive mechanism in an online community are summarized in Table 1.

What follows from this parallel? User modeling researchers may focus their attention on incentive mechanism design and community modeling as a more general case of adaptation and user-modeling. There exists already some work on group user modeling (UMUAI special issue on Group User Modeling in 2006). While the design 
Table 1 Parallels between personalization mechanism and incentive mechanism

\begin{tabular}{|c|c|}
\hline $\begin{array}{l}\text { Personalization mechanism in user-adaptive } \\
\text { system (micro-level) }\end{array}$ & $\begin{array}{l}\text { Incentive mechanism in an online } \\
\text { community (macro-level) }\end{array}$ \\
\hline User model: & Community model: \\
\hline $\begin{array}{l}\text { Individual user's preferences, interests, } \\
\text { ratings, knowledge, goals... }\end{array}$ & $\begin{array}{l}\text { Community participation, represented } \\
\text { according to a certain set of metrics } \\
\text { Individual models: } \\
\text { Individual participation represented } \\
\text { according to certain metrics }\end{array}$ \\
\hline Purpose of adaptation: & Purpose of adaptation: \\
\hline $\begin{array}{l}\text { Optimizing system behavior towards the } \\
\text { individual user } \\
\text { Recommending content of interest } \\
\text { for the user, } \\
\text { Adapting interface to the preferences / } \\
\text { level of knowledge/experience/current } \\
\text { goal of the user, } \\
\text { Stimulating reflection in user,... }\end{array}$ & $\begin{array}{l}\text { Optimizing system behavior with respect } \\
\text { to all the users in the system } \\
\text { Increasing the number and quality of } \\
\text { user contributions, } \\
\text { Binding the users in social ties, } \\
\text { Enticing users to commit to a } \\
\text { common goal, } \\
\text { Making the community } \\
\text { self-sustainable, ensuring growth } \\
\text { and stability... }\end{array}$ \\
\hline Adaptation interventions: & Adaptation interventions: \\
\hline $\begin{array}{l}\text { Showing recommendations, sorting list of } \\
\text { search results, reducing complexity of } \\
\text { interface or text, visual signaling,... }\end{array}$ & $\begin{array}{l}\text { Providing rewards for particular } \\
\text { actions (individually weighted), } \\
\text { Visualizing the community adaptively } \\
\text { to emphasize particular incentives }\end{array}$ \\
\hline
\end{tabular}

of user adaptive systems is often guided by insights from psychology, esp. cognitive psychology (in the case of adaptive learning environments), the design of incentive mechanisms is guided by theories from social psychology, organizational science and behavioral economics. Evaluation methodologies used in the area of user-adaptive systems design will likely have to be modified to be applicable in evaluating incentive mechanisms. The reason is that it is extremely hard to do controlled studies in online communities. The effect of the incentive mechanisms depends on the stage of the lifetime of the community (Lampe et al. 2010) in which they are applied. While studies involving large established communities are relatively easy to do (of course, if the researchers have access to data-sets from such communities), they are of relatively lesser value for practice, since the impact of incentives on an already established and active community is not so vital, as it is in new communities that are just starting. Attracting sufficient number of participants for experiments is harder since there aren't many users of the community at all. Yet the benefits of a mechanism that is effective in the early phase are much larger.

\subsection{Combining different incentive mechanisms in one system}

It seems logical that incentive mechanisms need to be personalized, because every person has different motivations, depending on personality, gender, age, education, 
stage in life, cultural background, interests, priorities, etc. As we saw in Sect. 2, most existing incentive mechanisms are not personalized. Even in the adaptive rewards version of Comtella (Cheng and Vassileva 2006) where the weights of different activities (i.e. the rewards) depended on a model of the user's previous contributions, the mechanism as a whole was still the same for all users, geared towards earning reputation, status and power in the community.

There is a good reason for having just one incentive mechanism in a community: designing an incentive mechanism is like making the rules of a game. Normally, all the players in a game are bound to follow the same rules. However, if the game is complex enough, it has many rules and some players may choose to follow mostly some of the rules, while not violating the others. For example, in a massive multiplayer game, like World of Warcraft (WoW) players can choose different roles and follow different goals and rules (Nardi and Harris 2006). Similarly, there may be several incentive mechanisms embedded in a community, e.g. one targeting the people who are motivated by reputation, another one-for people mostly motivated by power, and a third one-for people motivated by building balanced relationships with other users. While the resulting system will not be necessarily "personalized", it will provide an opportunity for users to choose and pursue their intrinsic personal motivations and set their goals, accordingly. Yet the introduction of different mechanisms in the same system is not straightforward. Interactions between different incentive mechanisms can lead to mutual cancelling out of their motivational effects, as some studies in Behavioural Economics show (Ariely 2008). The investigation of the motivational effects of different incentives, their combinations and side-effects is currently an active area of research in behavioral economics. Online communities design can contribute to this research by providing a test-bed for implementing mechanisms according to certain theories and observing how their effects play out in the community.

Social visualization is a good candidate for personalization. Erickson (2003) postulates that "Everyone sees the same thing: no customization" for Social Visualization, to ensure that the community has a common stage of action where everyone can observe everyone else, and social norms can get established. However, another one of his postulates states that the visualization does not need to show exact data, and that some exaggeration or the opposite can be justified depending on the goal. If the user is known to be competitive, a default social visualization encouraging social comparison and emphasizing the difference between the user and her peers in the dimensions of desirable action will be probably a more effective motivator, than a social visualization encouraging social bond and reciprocation. Yet, following the other Erickson's principles, the user should still have access to the other social visualizations, if multiple alternative ones have been developed to motivate different types of users.

\subsection{Bridging open user modeling and social visualization}

Parallels exist also between the area of open user modeling and social visualization (see Table 2). Open learner modeling (Bull et al. 2007), has been an active direction 
Table 2 Parallels between open user modeling and social visualization

\begin{tabular}{ll}
\hline Open user modeling, interaction analysis, & Social visualization \\
social network analysis & \\
\hline User model: & Community model: \\
Individual user's preferences, interests, likes, & Community participation, represented \\
dislikes, knowledge, goals & according to a certain set of metrics \\
User utterances & Individual models: \\
Communication acts between users & Individual participation represented \\
& according to certain metrics \\
Purpose: & Purpose of adaptation: \\
Stimulating reflection in user, & Stimulating social awareness \\
Recommending content of interest, focusing & Creating a stage for social events to happen \\
attention on important items to learn & and social norms to emerge \\
Informing teacher/moderator/manager about & Ultimately, increasing motivation, \\
the class/group/team's state. & engagement, participation \\
Adaptation interventions: & Adaptation interventions: \\
Emphasizing important areas according to & Visualizing the community adaptively to \\
certain criteria & emphasize particular incentives \\
\hline
\end{tabular}

in user modeling for 15 years now. Most of the works in this area focus on one-to-one systems aiming to open the learner model to the learner. With some notable exceptions, e.g. (Mazza and Dimitrova 2007; Ullman and Kay 2007), not much has been done so far for multi-user systems, like collaborative learning systems, e-learning systems and online learning communities.

The area of group or community modeling is still young. Group models can represent interactions among members of a group, individual contributions or relationships, or collaboration activities, stages, phases and processes (Soller 2007). Just like opening up individual models, opening group models to the users offers many advantages. It can help learners reflect on their progress in the group context, understand the problems others face. By externalizing the social model of the group, certain social norms are enforced and certain user behaviors are observed (Vassileva and Sun 2007, 2008).

In the area of Computer Supported Collaborative Learning (CSCL) and Computer Supported Collaborative Work (CSCW), a stream of research on Interaction Analysis finds patterns in the interactive/ collaborative activities in the group and represents the results in an appropriate way to the teacher/moderator. Researchers in CSCW from the Human-Computer Interaction community have produced a stream of work on social visualization, aimed at revealing a view of the other users and their activities in the community to the users, so that they can self-regulate their behaviors accordingly, mostly with respect to synchronizing their activities. In fact, these researchers are doing open group modeling.

A generalization of Interaction Analysis in the context of larger networks and communities is called Social Network Analysis (Spiliopoulu and Falkowsky 2007; Paliouras 2012). It has emerged as an area with a similar goal, to analyze data from user interactions and create maps of user relationships to inform managers, community moderators, teachers and users themselves. Typically data-mining techniques are 
applied in the analysis of large data-sets containing interaction data from online communities (e.g. discussion forums or blogs), organizations (e.g. organizational email archives), or collaboration networks (e.g. from large archives of co-authored papers).

There is an obvious link between the areas of Interaction Analysis, Community Visualization and Open User/Learner Modeling and the researchers working in these areas can learn from the experience of the others. For example, the question of how to represent visually the information from the user/group model or the results of interaction analysis in a way that it is understandable and useful is common for all these areas. Also common is the fundamental question which data to open (visualize), which depends on the goals set for the community or organization by its owner or manager (e.g. teacher, moderator, funding agency, etc.).

\subsection{Incentive mechanism design and self-directed learning}

With the availability of vast user-generated repositories of learning materials, many see the future of education in self-directed, life-long learning (Collins and Halverson 2009). The main problem becomes to motivate the learner to explore the available resources, participate in learning communities, and to maintain her level of motivation until she achieves her goals. This makes self-directed learning a particularly interesting application area to deploy and evaluate persuasive interventions and incentive mechanisms. Intelligent Tutoring Systems researchers have leveraged increased engagement and learning by incorporating game-like features (e.g. in Quest to Learn-www.q21. org) within learning environments (Burleson 2005; Jackson et al. 2009; Rowe et al. 2010). However, instructional planning on a macro-level has not been approached as a problem of persuasive intervention design or incentive mechanism design. Using concept maps of the subject area (Sosnovsky and Dicheva 2010) and AI planning techniques, paths for achieving particular learning goals can be generated. Previous work on course sequencing and dynamic courseware generation (Vassileva and Deters 1998; Brusilovsky and Vassileva 2003) can be used as a basis to inform content goal generation with integrated rewards to generate personalized learning plans. They will appear to the learner as paths of discovery in a game space, on a meta-level, creating curriculum-like structures of learning challenges, augmented with incentives (e.g. reputation or status-based, or credential-based), for achieving these challenges, adapted to the motivations (e.g. intrinsic, extrinsic, social) that might exist in the individual learner.

\section{Conclusions}

An important issue that was not discussed in this paper yet is the ethics of motivating people to stimulate particular behaviours. While it was implicitly assumed that motivational and incentive mechanisms are designed for "good" purposes, nothing prevents their exploitation for commercial purpose (we are already seeing very high interest in gamification from companies), and for darker purposes. One possible response (Kraut and Resnick, forthcoming) is that any design, whether it has explicit purpose to motivate or not, is motivational to a certain degree, since it shapes the user's experience 
and interaction. So it is better to be aware of this fact, and to responsibly include motivation in the design.

Motivating users to pursue particular goals or behaviors has gained importance in several different research areas:

- the design of online community infrastructures requires building incentives for participation in the interaction with the user, to deal with the cold start problem and to ensure sustainability for the communities.

- the design of persuasive systems aimed to help motivate users to adopt healthy lifestyles relies on understanding how to motivate users.

- the design on learning environments seeks to tap into the underlying learner motivations.

User modeling can play a key role in all these areas, since motivation is always personal. The design if incentive mechanisms can include personalized rewards and can adapt the rewards offered to the benefit of both the user and the entire community. There are interesting challenges lying ahead for user modeling researchers: investigating further how insights from theories of motivation and participation from the area of social sciences can be applied to guide the design of incentive mechanisms, how to adapt the motivational approach to the individual without disturbing the effects of the general incentive mechanism in the community, how to create models of groups and communities that can support adaptive incentive mechanisms, how to design open group models and social visualizations with particular motivational purpose.

\section{References}

Ajzen, I.: The theory of planned behavior. Org. Behav. Hum. Decis. Process. 50, 179-211 (1991)

Ariely, D.: Predictably irrational: the hidden forces that shape our decisions. HarperCollins, Noida (2008)

Bandura, A.: Self-efficacy: the exercise of control. Worth Publishers, New York (1997)

Berkovsky, Sh., Coombe, M., Freyne, J., Bhandari, D., Baghaei, N.: Physical activity motivating games: virtual rewards for real activity. International conference on human factors in computing systems, CHI'2010, pp. 243-252. Atlanta (2010)

Bozarth, J.: An interview with Sebastian deterding, eLearn, vol. 2011, No. 7. ACM Press, New York (2011)

Bretzke, H., Vassileva, J.: Motivating cooperation in peer to peer networks, Proceedings User Modeling UM03, June 22-26, pp. 218-227, Springer Verlag LNCS 2702, Johnstown (2003)

Brusilovsky, P., Vassileva, J.: Course sequencing techniques for large scale web-based education. Int. J. Contin. Eng. Educ. Life-Long Learning 13(1/2), 75-94 (2003)

Bull, S., Dimitrova, V., McCalla, G.: Open learner models: research questions. Int. J. Artif. Intel. Educ. 17(2), 83-87 (2007)

Burleson, W.: Opportunities for creativity, motivation, and self-actualization in learning systems. Int. J. of Human-Computer Stud. 63(4-5), 436-451 (2005)

Chen, Y., Harper, F.M., Konstan, J., Li Sh. X.: Social comparisons and contributions to online communities: a field experiment on movieLens. Computational Social Systems and the Internet 2007, Daghstuhl Seminar Proceedings (2007)

Cheng, R., Vassileva, J.: Adaptive reward mechanism for sustainable Online learning community. In: Proceedings Artificial Intelligence in Education (AIED’2005), IOS Press, pp. 152-159 Amsterdam, The Netherlands, July 18-22 (2005a)

Cheng, R., Vassileva, J.: User motivation and persuasion strategy for peer-to-peer communities. Proceedings HICSS'2005 (Mini-track on online communities in the digital economy/emerging technologies), Hawaii, IEEE Press. (CD ROM proceedings) (2005b)

Cheng, R., Vassileva, J.: Design and evaluation of an adaptive incentive mechanism for sustained educational online communities. User Model. User-Adapt. Interact. 16(2/3), 321-348 (2006) 
Cialdini, R.B.: The science of persuasion. Sci. Am. 284, 76-81 (2001)

Colineau, N., Paris, C.: Can beneficial habits be induced through reflection. Proceedings of Workshop on User Models for Motivational Systems: the affective and the rational routes to persuasion, with UMAP'2010, Hawaii (2010)

Collins, A., Halverson, R.: Rethinking education in the age of technology: the digital revolution and schooling in America. Teachers College Press, New York (2009)

Consolvo, S., McDonald, D., Landay, J.: Theory-driven design strategies for technologies that support behaviour change in everyday life. Proceedings of the SIGCHI Conference on Human Factors in Computer Systems (CHI'2009), pp. 405-414, ACM Press, Boston (2009)

Crumlish, Ch., Malone, E.: Designing social interfaces. O'Reilly, Chatsworth (2010)

Deci, E.L., Ryan, R.M.: Intrinsic motivation and self-determination in human behavior. Plenum, New York (1985)

Deterding, S., Dixon, D., Khaled, R., Nacke, L.E.: From Game Design Elements to Gamefulness: Defining "Gamification". In: Mindtrek 2011 Proceedings, ACM Press, Tampere (2011a)

Deterding, S., Khaled, R., Nacke, L., Dixon, D.: Gamification: Toward a Definition. CHI 2011 Gamification Workshop Proceedings, Vancouver (2011b)

Erickson, T.: Designing visualizations of social activity: Six claims. Proceedings of the human factors in computing systems, New Horizons, Proceeings of the SIGCHI conference on human factors in computer systems (CHI' 2003), pp. 846-847, ACM Press, Fort Lauderdale (2003)

Farzan, R., Dimicco, J., Millen, D., Dugan C., Geyer, W, Brownholtz, B.: Results from deploying a participation incentive mechanism within the enterprise. Proceedings of the SIGCHI conference on human factors in computer systems (CHI'2008), ACM Press, Florence (2008)

Fehr, E., Kirchsteiger, G., Riedl, A.: Gift exchange and reciprocity in competitive experimental markets. Eur. Econ. Rev., Elsevier 42(1), 1-34 (1998)

Festinger, L.: A theory of social comparison processes. Hum. Relations 7(2), 117-140 (1954)

Fogg, B.J.: Persuasive technology. Morgan Kaufmann, California (2003)

Freyne, J., Berkovsky, Sh.: Intelligent food planning: personalized recipe recommendation. Proceedings of the intelligent user interfaces (IUI 2010), pp. 321-324, Hong Kong (2010)

Golle, P., Leyton-Brown, K., Mironov, I.: Incentives for sharing in peer-to-peer networks, ACM conference on electronic commerce (EC-2001), pp. 264-267, Tampa (2001)

Greer, J., McCalla, G., Vassileva, J., Deters, R., Bull, S., Kettel, L.: Lessons learned in deploying a multiagent learning support system: the I-Help experience. Proceedings of AI in education AIED'2001, San Antonio, pp. 410-421, IOS Press, Amsterdam (2001)

Greer, J., McCalla, G., Cooke, J., Collins, J., Kumar, V., Bishop, A., Vassileva, J.: The intelligent helpDesk: supporting peer help in a university course. In: Goettl, B., Halff, H., Redfield, C., Shute, V. (eds.) Proceedings Intelligent Tutoring Systems, ITS'98, San Antonio, LNCS No. 1452, pp. 494-503, Springer Verlag, Berlin (1998)

Harper, F.M., Li, Sh.X., Chen, Y., Konstan, J.: Social comparisons to motivate contributions to an online community. Proceedings Persuasive 2007, pp. 148-159, Palo Alto (2007)

Hsieh, G., Kraut, R.E., Hudson, S.: Why pay. Exploring how financial incentives are used for questions and answers. Proceedings ACM SIGCHI conference on human factors in computing systems. CHI'10, Atlanta, ACM Press, New York (2010)

Jackson, G.T., Boonthum, C., McNamara, D.S.: iSTART-ME: Situating extended learning within a gamebased environment. Proceedings of the workshop on intelligent educational games with International Conference on AI and education AIED'2009, Brighton (2009)

Jones, Q., Rafaeli, S.: User population and user contributions to virtual publics: a systems model. Proceedings of the international ACM SIGGROUP conference on supporting group work, pp. 239-248, Phoenix, Arizona (1999)

Katz, E., Haas, H., Gurevitch, M.: On the use of the mass media for important things. Am. Sociolog. Rev. 38(2), 164-181 (1973)

Khaled, R., Barr, P., Noble, J., Biddle R.: Investigating social software as persuasive technology. Proceedings Persuasive 2006, Eindhoven, The Netherlands. LNCS 3962, pp. 104-107, Springer (2006)

Kim, A.J.: Community building on the web: secret strategies for successful online communities. Peachpit Press, Berkeley (2000)

Kimani S, Sh. Berkovsky, Smith, J. Freyne, N. Baghaei, D. Bhandari: Activity awareness in family-based healthy living online social networks. Proceedings of the intelligent user interfaces, IUI 2010, pp. 337-340, Hong Kong (2010) 
Kostuik K., Vassileva, J.: (1999) Free market control for a multi-agent based peer help environment. In: Proceedings of the workshop on agents for electronic commerce and managing the internet-enabled supply chain, held in association with the 3rd international conference on autonomous agents (Agents '99), Seattle, May 1-5 (1999)

Kraut, R.E., Resnick, P.: Evidence-based social design: Mining the social sciences to build online communities. MIT Press, Cambridge (in press)

Lampe, C., Wash, R., Velasquez, A., Ozkaya, E.: Motivations to participate in online communities. Proceedings of the ACM SIGCHI conference on human factors in computing systems (CHI'2010) pp. 1927-1936, Atlanta (2010)

Latham, G., Locke, E.: Building a practically useful theory of goal setting and task motivation. Am. Psycholog. 57(9), 707-709 (2002)

Lepper, M.R., Greene, D., Nisbett, R.E.: Undermining children's intrinsic interest with extrinsic rewards: A test of the overjustification hypothesis. J. Personal. Soc. Psychol. 28(1), 129-137 (1973)

Lin, J.J., Mamykina, L., Lindtner, S., Delajoux, G., Strub, H.B.: Fish'n'Steps: Encouraging physical activity with an interactive computer game, Proceedings of the international conference on ubiquitous computing, UbiComp'06, pp. 261-278, Orange County (2006)

Ling, K., Beenen, G., Ludford, P.J., Wang, X., Chang, K., Li, X., Cosley, D. Frankowski, D., Terveen, L., Rashid, A., Resnick, P., Kraut, R.: Using social psychology to motivate contributions to online communities. J. Comput. Mediat. Commun. 10(4) (2005)

Mao, Y., Vassileva, J., Grassmann, W.: (2007) A System Dynamics Approach to Study Virtual Communities. In: Proceedings IEEE HICSS'07 mini-track on Virtual Communities. Big Island, Hawaii, Jan 2-5 (2007)

Mazza, R., Dimitrova, V.: CourseVis: a graphical student monitoring tool for supporting instructors in web-based distance courses. Int. J. Hum.-Comput. Stud. 65(2), 125-139 (2007)

McDonald, P.: Game over? When play becomes mechanical. Planning in high heels blog, Nov. $25,2010$. Available online at (last accessed on April 27, 2010) http://planninginhighheels.com/2010/11/25/ game-over-when-play-becomes-mechanical/ (2010)

Mudgal, C., Vassileva, J.: Bilateral negotiation with incomplete and uncertain information, in CIA'2000. In: Klusch and Kershberg (eds.) Proceedings of the workshop on cooperative information agents, Springer LNAI 1860, pp. 107-118 (2000)

Munson, S., Lauterbach, D., Newman, M., Resnick, P.: Happier Together: Integrating a wellness application into a social network site. Proceedings of the Persuasive 2010, Copenhagen, Denmark (2010)

Nardi, B., Harris, J.: Strangers and friends: Collaborative play in world of warcraft. Proceedings ACM conference on computer supported cooperative work, CSCW'2006, pp. 149-138, Banff (2006)

Nguyen, H., Masthoff, J.: Developing personalised digital behaviour interventions: A multi stage process Proceedings of the workshop on user models for motivational systems, with UMAP'2010, pp. 43-52 (2010)

Paliouras, G.: Discovery of web user communities and their role in personalization. User Model. User-Adapt. Interact. 22(1-2), 151-175 (2012)

Porter, J.: Designing for the social web. Berkley, New Riders (2008)

Radia, S.: Digital communities can learn from "Leading Clever People", BBH labs blog, Nov 23, 2010. Available online at (last accessed on April 27, 2011) http://bbh-labs.com/digital-communitiescan-learn-from-leading-clever-people

Rafaeli, S., Raban, D.R., Ravid, G: Social and economic incentives in google answers. In: Sangeetha, K., Sivarajadhanavel, P. Google's growth, a success story, pp. 150-161. ICFAI University Press, Hyderabad (2007)

Raghavun, K., Vassileva, J.: Visualizing reciprocity to motivate participation in an online community, Proc. 5th IEEE International Conference on Digital Ecosystems and Technologies Conference (DEST) May 31-June 3, pp. 89-94, IEEE Press, Daejeon, Korea (2011)

Rashid, A., Ling, K., Kraut, R., Riedl, J.: Motivating participations by displaying the value of contribution. Proceedings of the ACM SIGCHI conference on human-factors in computing systems ( CHI 2006). Montreal, QC, Canada, New York: ACM Press, 955 - 958 (2006)

Reiss, S.: Multifaceted nature of intrinsic motivation: the theory of 16 basic desires. Rev. General Psychol. 8(3), 179-219 (2004)

Ren Y., Kraut, R.: A simulation of designing online community: member motivation, contribution, and discussion moderation, (unpublished), Available online (manuscript last accessed on Nov 10, 2010). 
http://www.cs.cmu.edu/kraut/RKraut.site.files/articles/ren07-SimulatingOnlineCommunit-v4.6-rek. pdf

Ren, Y., Kraut, R.E., Kiesler, S.: Applying common identity and bond theory to the design of online communities. Org. Stud. 28(3), 379-410 (2007)

Rowe, J., Shore, L., Mott, B., Lester, J.: Integrating learning and engagement in narrative-centered learning environments. Proceedings of the Intelligent Tutoring Systems (ITS'2010), pp. 166-177, Pittsburgh (2010)

Soller, A.: Adaptive support for distributed collaboration. In: Brusilovsky, P., Kobsa, A., Neidl, W. The adaptive web, LNCS 4321, Springer-Verlag, Berlin, Heidelberg (2007)

Sosnovsky, S., Dicheva, D.: Ontological technologies for user modelling. Int. J. Metadata, Semantics and Ontologies 5(1): Inderscience, pp. 32-71 (2010)

Spiliopoulu, M., Falkowsky, T.: Users in volatile communities: studying active participation and community evolution. Proceedings user modeling UM'2007, Corfu, pp. 47-56, Springer Verlag LNCS 4511 (2007)

Suls, J., Martin, R., Wheeler, L.: Social comparison: why, with whom, and with what effect? Curr. Direct. Psychol. Sci. 159-163 (2002)

Sun, L., Vassileva, J.: Social visualization encouraging participation in online communities, Proceedings of the 12th International Workshop on Groupware, CRIWG'2006, Valladolid, Spain, pp. 349-363, Springer Verlag LNCS 4154 (2006)

Turner, J.C., Hogg, M.A., Oakes, P.J., Reicher, S.D., Wetherell, M.S: Rediscovering the social group: a self-categorization theory.573-595 Blackwell, Oxford (1987)

Turner, J.C.: Social categorization and the self-concept: a social cognitive theory of group behavior. In: Lawler E.J. (ed.) Advances in group processes: theory and research, vol. 2. pp. 77-122, JAI Press, Greenwich (1985)

Ullman, A.J., Kay, J.: WikiNavMap: a visualisation to supplement team-based wikis. Extended Abstracts of the SIGCHI conference on human factors in computer systems (CHI'2007) pp. 2711-2716, ACM Press, San Jose (2007)

Vassileva, J.: Harnessing P2P power in the classroom. In: Lester, J., Vicari, R., Paraguacu, F. (eds.) Proceedings of the intelligent tutoring systems, ITS'2004,. Lecture Notes in Computer Science No. 3220, pp. 305-314 (2004)

Vassileva, J., Deters, R.: Dynamic courseware generation on the WWW. Br. J. Educ. Technol. 29(1), 5-14 (1998)

Vassileva, J., Sun, L.: Using community visualization to stimulate participation in online communities. E-Serv. J. 6(1), 3-40 (2007)

Vassileva, J., Sun, L.: Evolving a social visualization design aimed at increasing participation in a class-based online community. Int. J. Cooper. Inform. Syst. (IJCIS) 17(4), 443-466 (2008)

Vassileva, J., Greer, J., McCalla, G., Deters R., Zapata, D., Mudgal, C., Grant, S.: A multi-agent approach to the design of peer-help environments. In: Proceedings AI in Education AIED’99, pp. 38-45, Le Mans (1999)

Vassileva, J. et al.: Supporting peer-to-peer user communities. In: Meersman, R., Tari, Z. (eds.) On the move to meaningful internet systems 2002: CoopIS, DOA, and ODBASE coordinated international conferences proceedings, Irvine, 29 Oct-1 Nov 2002, LNCS 2519, pp. 230-247. Springer Verlag, Berlin, Heidelberg (2002)

Wang, Y., Vassileva, J.: Trust and reputation model in peer-to-peer networks. Proceedings of the IEEE conference on peer-to-peer computing (P2P'2003) pp. 150-157, IEEE Press, Linkoeping (2003)

Wang, Y., Vassileva, J.: Trust-based community formation in peer-to-peer file sharing networks. Proceedings of IEEE/WIC/ACM international conference on web intelligence (WI 2004), Sep 20-24, Beijing (2004)

Webster, A.S., Vassileva, J.: Visualizing personal relations in online communities. Proceedings of the adaptive hypermedia and adaptive web-based systems (AH'2006), June 21-23, pp. 223-233, Springer LNCS 4018, Dublin (2006)

Winoto, P., McCalla, G., Vassileva, J.: Non-monotonic-offers bargaining protocol. J. Auto. Agents MultiAgent Syst. 11(1), 45-67 (2005)

Wu, M.: The gamification backlash + two long term business strategies, Lithosphere - Michael Wu's blog (last accessed Oct 13, 2011) http://lithosphere.lithium.com/t5/Building-Community-the-Platform/ The-Gamification-Backlash-Two-Long-Term-Bussiness-Strategies/ba-p/30891 


\section{Author Biography}

Julita Vassileva is a professor of computer science at the University of Saskatchewan, Canada and one of the directors of the MADMUC Lab. Dr. Vassileva received her PhD in Mathematics (Cybernetics and Control Theory) from the University of Sofia/ Bulgarian Academy of Sciences. Her research areas involve human issues in decentralized software environments: user modeling and personalization, designing incentive mechanisms for encouraging user participation and facilitating trust in decentralized software applications, such as online communities, social networks, open learning environments, multi-agent systems, and peer-to-peer systems. 